\title{
BLPA : Bayesian Learn-Predict-Adjust Method for Online Detection of Recurrent Changepoints
}

\author{
Maslov, Alexandr \\ Institute of Electrical and Electronics Engineers \\ 2017
}

Maslov , A, Pechenizkiy , M , Pei , Y, Zliobaite , I, Shklyaev , A, Kärkkäinen, T \& Hollmén , J 2017 , BLPA : Bayesian Learn-Predict-Adjust Method for Online Detection of Recurrent Changepoints . in IJCNN 2017 : The International Joint Conference on Neural Networks . Proceedings of ... International Joint Conference on Neural Networks, Institute of Electrical and Electronics Engineers , Piscataway, NJ , pp. 1916-1923, International Joint Conferenceon Neural Networks , Anchorage , United States, 14/05/2017 . https://doi.org/10.1109/IJCNN.2017.7966

http://hdl.handle.net/10138/307576

https://doi.org/10.1109/IJCNN.2017.7966085

unspecified

acceptedVersion

Downloaded from Helda, University of Helsinki institutional repository.

This is an electronic reprint of the original article.

This reprint may differ from the original in pagination and typographic detail.

Please cite the original version. 


\title{
BLPA: Bayesian Learn-Predict-Adjust Method for Online Detection of Recurrent Changepoints
}

\author{
Alexandr Maslov*, Mykola Pechenizkiy*, Yulong Pei*, \\ Indrė Žliobaitè ${ }^{\S}$, Alexander Shklyaev ${ }^{\ddagger}$, Tommi Kärkkäinen ${ }^{\dagger}$ and Jaakko Hollmén ${ }^{\S}$ \\ * Eindhoven University of Technology, Dept. of Mathematics and Computer Science, The Netherlands \\ Email: \{a.maslov, m.pechenizkiy, y.pei.1\}@tue.nl \\ $\dagger$ University of Jyväskylä, Department of Mathematical Information Technology, Finland \\ Email: tommi.karkkainen@jyu.fi \\ $\ddagger$ Lomonosov Moscow State University, Dept. of Mechanics and Mathematics, Russia \\ Email: ashklyaev@gmail.com \\ $\S$ Aalto University, Dept. of Computer Science, Finland \\ Email: zliobaite@gmail.com, Jaakko.Hollmen@aalto.fi
}

\begin{abstract}
Online changepoint detection is an important task for machine learning in changing environments. Presence of noise that can be mistaken for real changes makes it difficult to develop an effective approach that would have a low false alarm rate and being able to detect all the changes with a minimal delay. In this paper we study how performance of popular Bayesian online detectors can be improved in case of recurrent changes. Modeling recurrence allows us to anticipate future changepoints and predict their time locations. We propose BLPA, an efficient approach for inducing and integrating recurrence information in the streaming settings, and demonstrate its effectiveness in the experimental study on synthetic and real-world datasets.
\end{abstract}

\section{INTRODUCTION}

Online change detection is practically relevant in many domains, such as medicine, energy production, industrial processes monitoring [1]. In machine learning and data mining research areas change detection is often studied in the context of problem of concept drift happening due to changes in the underlying data distribution over time [2]. A popular approach for handling concept drift is to monitor data or model performance for changes and to adapt model using most recent data collected after the last detected change [3].

In this paper we consider a change detection task in a onedimensional univariate time series data streams. Further in the text we denote a univariate vector of observations either as $\left\langle x_{i}\right\rangle_{i=1}^{n}$ or as $\boldsymbol{x}_{1: n}$, i.e.

$$
\boldsymbol{x}_{1: n} \equiv\left\langle x_{i}\right\rangle_{i=1}^{n} \equiv\left\langle x_{1}, \ldots, x_{n}\right\rangle
$$

Input to the change detector is a vector of observations $\left\langle x_{t}\right\rangle$ indexed by the timestamps $t \in \mathcal{T}$. Timestamps is an ordered vector of time moments $\mathcal{T} \equiv\left\langle t_{1}, \ldots, t_{T}\right\rangle$ when observations were taken with a constant sampling rate. Changepoint is a time moment when statistical properties of the data stream change significantly according to the predefined criteria. Changepoint is identified by the moment of time when it happened (further - 'time location of the change'). The sequence of changes is denoted as $\left\langle c_{i}\right\rangle_{i=1}^{k} \in \mathcal{T}$ and an individual change from this sequence as $c_{i}$. Changes should be detected online when the only information observed until current moment of time can be used for an analysis.

The top plot (A) in the Fig. 1 illustrates an example of the input signal with three changepoints in the mean value at the moments $\boldsymbol{c}_{1: 3}=\langle 5,10,14\rangle$. Change is usually detected with some time delay $\delta$. The change detection task is to detect changes $\boldsymbol{c}_{1: 3}$ with as small a possible delay $\delta$ while not alarming changes at any other time moments, i.e. avoiding false alarms as much as possible.

An event when the change was alarmed by the detector while there is actually no change is called False Positive (FP). Outliers and noisy changes in the input signal may cause FPs.

While the majority of existing change detection techniques focus on individual changepoint detection and assume that changepoints are not predictable, Fig. 11 illustrates use cases in which changes are expected to reappear over time. In this paper we focus on such setting, addressing the problem of detecting changes in noisy signals with recurrent changes.

Our approach (called BLPA method) is based on the hypothesis that if probability distribution of the time intervals between changepoints differs from the probability distribution of time intervals between outliers we can use this information to predict time locations of the changes and skip outliers and therefore achieve better TP/FP rates.

BLPA is a new online detection method. It extends the Bayesian Online Changepoint Detector (BD) proposed in [4] by embedding into it a Predictive Change Confidence Function 
(PCCF), which we introduced recently in [5], in order to predict future changepoints in the input data stream, adjust detector's settings dynamically and to reduce FP rate.

In short, BD detector works by recursively estimating posterior probability distribution $P\left(r_{t} \mid \boldsymbol{x}_{1: t}, \theta\right)$ of the run length variable $r_{t}$ which is a time since the last changepoint. Changepoint is an event when

$$
\underset{r_{t}}{\arg \max } P\left(r_{t} \mid \boldsymbol{x}_{1: t}, \theta\right)=0
$$

The posterior distribution is recalculated then every time a new measurement $x_{t}$ is observed using Bayes' theorem to update parameters of the distributions used to model data and the law of total probability

$$
P\left(r_{t} \mid \cdot\right)=\sum_{r_{t-1}} P\left(r_{t} \mid r_{t-1}, \cdot\right) P\left(r_{t-1} \mid \cdot\right)
$$

to consider all possible run's values in the past.

The prior probability of the change $P\left(r_{t}=0 \mid t\right)$ in BD detector is specified using the constant-value hazard rate $h$ which is an instant prior probability to observe a change and which is supposed to be known before the change detection process starts. The uniform non-informative prior does not hold enough information to distinguish outliers and noisy changes from the changepoints. We improve performance of the $\mathrm{BD}$ detector by using an informative prior distribution in a form of the PCCF function which parameters are the average time interval $\mu$ between consecutive changepoints $\left\langle c_{i}-c_{i-1}\right\rangle$ and standard deviation $\sigma$. Given current estimate of $\mu$ and $\sigma$ PCCF gives a prior probability $\mathcal{P}(t \mid \mu, \sigma)$ to observe recurrent changepoint at time $t$. During the change detection process parameters of the BD detector are adjusted dynamically according the predictions in order to skip possible noisy changes in between changepoints. When a new changepoint is detected (or its location is provided by outer source) parameters $(\mu, \sigma)$ are updated using Bayesian rule and new prediction $\mathcal{P}\left(t \mid \mu_{\text {new }}, \sigma_{\text {new }}\right)$ is made.

The paper is organized as follows. In Section $I$ we review related works. In Section III we describe in detail how the Bayesian Change Detector proposed in [4] works. In Section IV we describe PCCF function used to predict recurrent changes. In Section $\mathrm{V}$ we describe the data model common for the input signal of observations $\left\langle x_{i}\right\rangle$ and for the time intervals between changepoints $\left\langle c_{i}-c_{i-1}\right\rangle$. In the Section VI we describe the BLPA algorithm which is a BD detector integrated with the PCCF function. In the Section VII we describe experimental results demonstrating improved performance of the BD detector when integrated with the PCCF.

\section{RELATED WORK}

While many change detection methods have been developed [1], [6] for offline and online settings, they typically assume that changes occur at random in time, and are independent from each other. In practice, however, in many industrial applications changes occur with some regularity (e.g. seasonality). Our BLPA approach captures this information from data, and utilizes it for improving the accuracy of a Bayesian online change detection.

In the Bayesian online change detector proposed in [4] and extended in [7] authors model time intervals between change points (run lengths) using the hazard rate. This approach allows to take into account recurrence by tuning single parameter, but it does not allow to distinguish outliers from changes which may appear between them. In [8] data stream volatility, defined as the rate of detected changes, is used to make detector more reactive. We concentrate on the problem of improving change detection by predicting time locations of the changes in the future in order to better distinguish outliers from real changes.

In $\mathrm{BD}$ [4] the hazard rate is a constant value assumed to be known in advance. This is not a realistic assumption and this problem has been addressed in [9] where authors proposed an on-line inference procedure to estimate $h$ parameter for the case if hazard rate is unknown and can itself undergo changes while new data arrives. In [10] authors proposed an algorithm which can detect and locate changepoints simultaneously using Bayesian statistics approach. In [11] authors use Gaussian Process model to compute predictive distribution $p\left(x_{\mathbf{n e w}} \mid x_{\mathbf{o l d}}\right)$.

Our method is different from these ones because we combine change detection and prediction tasks. We add a second layer (PCCF function) on top of the change detection algorithm allowing to predict future recurrent changes and adjust detectors settings dynamically. This second layer is a change detector itself in the sense that it automatically incorporates changes in underlying distribution of the time intervals between recurrent changes.

In our previous work [5] we demonstrated how to integrate PCCF with the very naive threshold based detector in a heuristic way. The BLPA method we propose here is a more advanced. It integrates PCCF natively into the BD detector using Bayesian statistics framework. BLPA updates both parameters of BD and PCCF sequentially, detects changes, predicts future changes and adjusts parameters of the BD detector according to the predictions in order to skip noisy changes and outliers while detecting changes of interest.

A few other and more remote lines of work relate to our approach via attention to recurrent concept drift [12], [13], [14], predictability of concept drift [15], or change detection with delayed labeling [16]. These approaches are specific to handling concept drift, while our focus is on generic online change detection and its accuracy.

\section{ONLINE BAYESIAN Change Detector (BD)}

In this section we describe the Bayesian Online Changepoint Detector proposed in [4]. As we mentioned - to model time occurrences of the changes authors introduce a latent variable run length $r_{t}$ which is the number of time steps since the most recent change. In Fig. 1 plot (A) you can see an illustrating example of the input signal and corresponding run values on plot (B).

On each time step there are two possibilities: either the run length increases $r_{t}=r_{t-1}+1$ or changepoint occurs $r_{t}=0$. 
The conditional prior $P\left(r_{t} \mid r_{t-1}\right)$ of the change is given by a constant-value hazard rate $h$ (Equation 11).

$$
p\left(r_{t} \mid r_{t-1}\right)= \begin{cases}1-h & \text { if } r_{t}=r_{t-1}+1 \\ h & \text { if } r_{t}=0\end{cases}
$$

The plot (C) in Fig. 1 1 illustrates the message-passing algorithm to compute prior probabilities of the changepoint at any time moment given the boundary condition $P\left(r_{1}=0\right)=1.0$ that change occurred at the moment $t=1$. Each node (circle) represents a hypothesis about the current run length value. From each node there is a solid line upwards depicting probability of increasing of the run on the next time step (no change) and a dashed line going downwards depicting probability of the change.

At each time step the probability of the changepoint is estimated by calculating posterior probability distribution of the run length value given the data so far observed (Equation 23.

$$
P\left(r_{t} \mid \boldsymbol{x}_{1: t}\right)=\frac{P\left(r_{t}, \boldsymbol{x}_{1: t}\right)}{P\left(\boldsymbol{x}_{1: t}\right)}
$$

The joint probability of the run length values and observed so far data can be sequentially computed using recursive procedure in Equation 3 as it is described in [4]:

$$
\begin{gathered}
P\left(r_{t}, x_{1: t}\right)=\sum_{r_{t-1}} P\left(r_{t}, r_{t-1}, x_{1: t}\right)= \\
\sum_{r_{t-1}} P\left(r_{t}, x_{t} \mid r_{t-1}, x_{1: t-1}\right) P\left(r_{t-1}, x_{1: t-1}\right)= \\
\sum_{r_{t-1}} P\left(r_{t} \mid r_{t-1}\right) P\left(x_{t} \mid r_{t-1}, x_{t}^{(r)}\right) P\left(r_{t-1}, x_{1: t-1}\right)
\end{gathered}
$$

where $x_{t}^{(r)} \equiv\left\langle x_{t-r+1}, \ldots, x_{t}\right\rangle$ is input data sub-interval associated with the run length $r$. Marginal predictive distribution of the new observation $x_{t}$ is computed using the sum rule:

$$
P\left(x_{t} \mid \boldsymbol{x}_{1: t-1}\right)=\sum_{r_{t}} P\left(x_{t} \mid r_{t}, \boldsymbol{x}_{t}^{(r)}\right) P\left(r_{t} \mid \boldsymbol{x}_{1: t-1}\right)
$$

\section{PCCF FUNCTION}

In this section we show how to compute PCCF function used to predict time locations of the recurrent changes in the future. We consider a discrete case when observations are obtained at the discrete time moments $\langle t\rangle_{t=1}^{T}$ with a constant sampling rate. Probability distribution for the discrete sets is defined using Probability mass function (Pmf). As mentioned earlier we assume that changes $r$-occur after 'approximately' equal time intervals. To model time intervals between consecutive changes $\left\langle c_{i}-c_{i-1}\right\rangle$ we use the Gaussian distribution assuming that standard deviation is small enough so that probability to observe the change $c_{i}$ before $c_{i-1}$ is extremely small.

Definition 1: Changes $\left\langle c_{i}\right\rangle_{i=1}^{k}$ are recurrent if

$$
p\left(c_{i+1}=t \mid \theta^{C}\right)=p\left(c_{1}=t-c_{i} \mid \theta^{C}\right),
$$

where $\theta^{C}=\left(\mu^{C}, \sigma^{C}\right), c_{1}$ is the time of the $1^{\text {st }}$ change, $c_{i}$ is the time of the $i^{\text {th }}$ change.

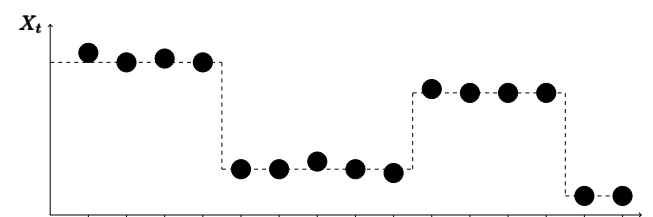

(A)
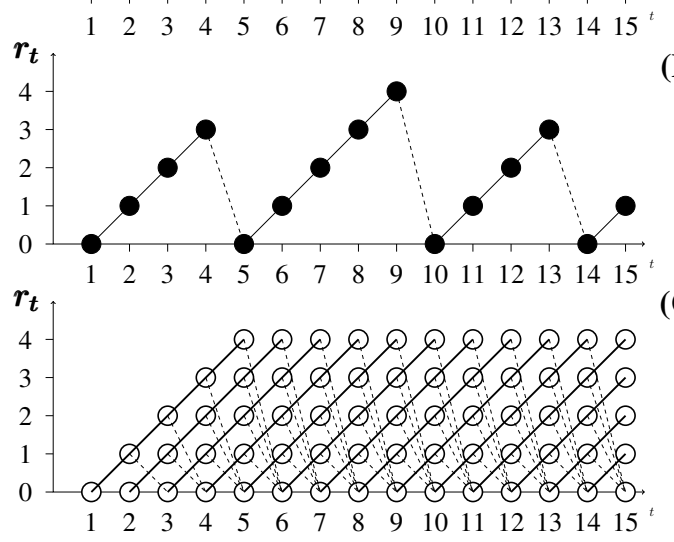

(C)

Fig. 1. The changepoint detection problem. (A): Input signal. (B): A particular realization of the run length path corresponding to the actual changepoints locations in the input signal. (C): Directed graph representing all possible run length paths. The figure is replicated from the illustration in [4].

This definition corresponds to the generative model defined by Equation 6 in which every next change $c_{i+1}$ happens after time intervals $\Delta$ which are samples from the Gaussain distribution $N\left(\mu^{C}, \sigma^{C}\right)$.

$$
c_{i+1}=c_{i}+\Delta, \text { where } \Delta \sim N\left(\mu^{c}, \sigma^{c}\right)
$$

To predict future changes we introduce the notion of the Predictive Change Confidence Function (PCCF ) [5].

Definition 2: PCCF is a Pmf defined on a discrete set of time moments $\langle t\rangle_{t=1}^{T}$ giving a probability to observe recurrent change $\forall c \in\left\langle c_{i}\right\rangle_{i=1}^{k}$ at the time moment $t$

$$
\mathcal{P}\left(c=t \mid \mu^{c}, \sigma^{c}\right)=\sum_{i=1}^{k} p\left(c_{i}=t \mid \mu^{c}, \sigma^{c}\right)
$$

where $p\left(c_{i}=t \mid \mu^{c}, \sigma^{c}\right)$ is a Pmf for an individual change $c_{i}$. It is important to note that change-events $\left\langle c_{i}\right\rangle$ are independent. Every $c_{i}$ can happen at any moment of time according to its individual Pmf $p\left(c_{i}=t \mid \mu^{c}, \sigma^{c}\right)$. Following the sum rule for total probability $\sqrt{1}$ in order to compute $\operatorname{Pmf}$ of $c_{i+1}$ we need to consider all possible time locations of $c_{i}$.

$$
p\left(c_{i+1}=t\right)=\sum_{\tau=i}^{t-1} p\left(c_{i+1}=t \mid c_{i}=\tau\right) p\left(c_{i}=\tau\right) .
$$

According to the definition 2 PCCF is a sum of individual Pmf's of the changes which might happen till current moment

$$
{ }^{1} P(x)=\sum_{y} P(x \mid y) p(y)
$$


of time

$$
\begin{aligned}
\mathcal{P}(t) & =\sum_{i=1}^{t} \sum_{\tau=i}^{t-1} p\left(c_{i+1}=t \mid c_{i}=\tau\right) p\left(c_{i}=\tau\right) \\
& =\sum_{i=1}^{t} \sum_{\tau=i}^{t-1} p\left(c_{1}=t-c_{i}\right) p\left(c_{i}=\tau\right)
\end{aligned}
$$

Right side of the Equation 8 is a convolution for the $\operatorname{Pmf} p\left(c_{1}\right)$ of the $1^{\text {st }}$ recurrent change and of the Pmf of the change $c_{i}$ computed in the previous step

$$
\begin{aligned}
p\left(c_{i+1}\right) & =\left(p\left(c_{1}\right) * p\left(c_{i}\right)\right)[\tau] \\
& =\sum_{\tau=1}^{t-1} p\left(c_{1}=t-\tau\right) p\left(c_{i}=\tau\right) .
\end{aligned}
$$

The convolution of two Gaussian distributions is also Gaussian distribution

$$
\left(p\left(x \mid \mu_{1}, \sigma_{1}\right) * p\left(x \mid \mu_{2}, \sigma_{2}\right)\right)=p\left(x \mid \mu_{1}+\mu_{2}, \sqrt{\sigma_{1}^{2}+\sigma_{2}^{2}}\right) .
$$

PCCF (Eq. 9] can be written as a t-fold convolution

$$
\mathcal{P}(t)=(\underbrace{p\left(c_{1}\right) * p\left(c_{1}\right) * \cdots * p\left(c_{1}\right)}_{\mathrm{t}})
$$

which is equivalent to the sum

$$
\mathcal{P}(t)=\sum_{l=1}^{t} \frac{1}{\sigma \sqrt{2 \pi l}} \exp \left(\frac{-(t-l \mu)^{2}}{2 l \sigma^{2}}\right) .
$$

The sum 13 describes renewal-reward process [17], [18]. Using the renewal theorem [17] we can calculate the limit of $\mathcal{P}(t)$ when $t \rightarrow \infty$

$$
L=\lim _{t \rightarrow \infty} \sum_{l=1}^{\infty} \frac{1}{\sigma \sqrt{2 \pi l}} \exp \left(-\frac{(t-\mu l)^{2}}{2 l \sigma^{2}}\right)=\frac{1}{\mu} .
$$

From Equation 14 follows that PCCF converges to the constant value uniform distribution for large $t$ values. Fig. 2 illustrates two PCCF functions (Equation 13) with parameters $(\mu=10, \sigma=2)$ and $(\mu=15, \sigma=3)$. Prior and posteriors for

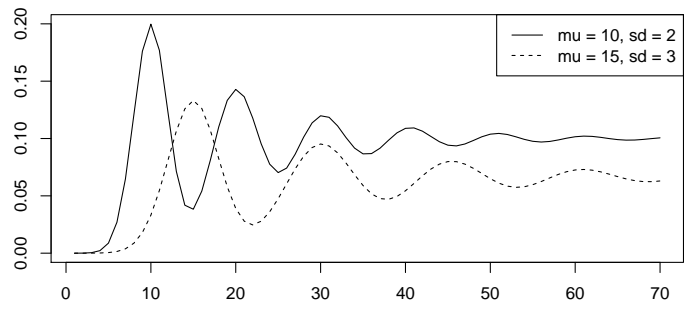

Fig. 2. An example of two Gaussian PCCF functions. The limits are $\frac{1}{\mu^{C}}$.

the PCCF's parameters are estimated and updated using the procedure described in Section $\mathrm{V}$ describing data model.

\section{DATA MODEL}

In this section we describe the data model which we use in BLPA. There are two streams of data to be analysed. The stream of input observations $\left\langle x_{t}\right\rangle$ and the stream of time intervals between changepoints $\left\langle c_{i}-c_{i-1}\right\rangle$. The first stream is used to detect changes and therefore to produce the second stream. The stream of changes maybe updated by the outer sources providing additional information about time location of the changes. E.g. there is a process running in parallel with the main detector which can run additional changedetection processes over collected data to identify locations of the changes in the past more precisely. The stream of changepoints is used to predict future changepoints in order to adjust detector's settings to achieve a better performance. Further, data $D$ is either input data stream of observations $\left\langle x_{t}\right\rangle$ or data stream of time intervals between consecutive changepoints $\left\langle c_{i}-c_{i-1}\right\rangle$. In this section we describe a data model for $D$ common both for input signal and sequence of time intervals between changepoints.

Data $D$ is assumed to be generated by a Gaussian distribution with an unknown mean and variance. We denote elements of $D$ by $\tilde{x}_{i} \in D$ with mean and variance $(\tilde{\mu}, \tilde{\sigma})$.

\section{A. Prior distributions.}

Following the notations in [19], we use a normal-gamma prior for $\tilde{\mu}$ and $\tilde{\sigma}$ :

$$
\begin{aligned}
\tilde{x}_{i} & \sim N(\tilde{\mu}, \tilde{\tau}), \quad \tilde{\tau}=(1 / \tilde{\sigma})^{2} \\
\tilde{\mu} & \sim N\left(\tilde{\mu}_{0}, \tilde{\kappa}_{0} \tilde{\tau}\right) \\
\tilde{\tau} & \sim \operatorname{Gamma}\left(\tilde{\alpha}_{0}, \tilde{\beta}_{0}\right)
\end{aligned}
$$

where $\left(\tilde{\alpha}_{0}, \tilde{\beta}_{0}, \tilde{\mu}_{0}, \tilde{\kappa}_{0}\right)$ are hyperparameters. The value $\tilde{\tau}$ is also named precision ${ }^{2}$. The likelihood of data $D=\left\langle\tilde{x}_{i}\right\rangle$ is

$$
P(D \mid \tilde{\mu}, \tilde{\tau})=\left(\frac{\tilde{\tau}}{2 \pi}\right)^{n / 2} \exp \left(-\frac{\tilde{\tau}}{2} \sum_{i=1}^{n}\left(\tilde{x}_{i}-\mu\right)^{2}\right)
$$

The joint conjugate prior for parameters $(\tilde{\mu}, \tilde{\tau})$ is the defined normal-gamma $(N G)$ distribution:

$$
\begin{aligned}
& P\left(\tilde{\mu}, \tilde{\tau} \mid \tilde{\mu}_{0}, \tilde{\kappa}_{0}, \tilde{\alpha}_{0}, \tilde{\beta}_{0}\right)=N\left(\tilde{\mu}_{0}, \tilde{\kappa}_{0} \tilde{\tau}\right) \operatorname{Gamma}\left(\tilde{\alpha}_{0}, \tilde{\beta}_{0}\right) \\
& =\frac{1}{Z} \tilde{\tau}^{1 / 2} \exp \left(-\frac{\tilde{\kappa}_{0} \tilde{\tau}}{2}\left(\tilde{\mu}-\tilde{\mu}_{0}\right)^{2}\right) \tilde{\tau}^{\tilde{\alpha}_{0}-1} e^{-\tilde{\tau} \tilde{\beta}_{0}} \\
& =\frac{1}{Z} \tilde{\tau}^{\tilde{\alpha}_{0}-1 / 2} \exp \left(-\frac{\tilde{\tau}}{2}\left[\tilde{\kappa}_{0}\left(\tilde{\mu}-\tilde{\mu}_{0}\right)^{2}+2 \tilde{\beta}_{0}\right]\right)
\end{aligned}
$$

where $Z=\frac{\Gamma\left(\tilde{\alpha}_{0}\right)}{\tilde{\beta}_{0}^{\tilde{\alpha}_{0}}}\left(\frac{2 \pi}{\tilde{\kappa}_{0}}\right)^{1 / 2}$ is the normalized factor.

\section{B. Posterior distributions}

The posterior can be derived as

$$
\begin{aligned}
P(\tilde{\mu}, \tilde{\tau} \mid D) & \propto P\left(\tilde{\mu}, \tilde{\tau} \mid \tilde{\mu}_{0}, \tilde{\kappa}_{0}, \tilde{\alpha}_{0}, \tilde{\beta}_{0}\right) P(D \mid \tilde{\mu}, \tilde{\tau}) \\
& \propto N\left(\tilde{\mu}_{n}, \tilde{\kappa}_{n} \tilde{\tau}\right) \operatorname{Gamma}\left(\tilde{\alpha}_{0}+n / 2, \tilde{\beta}_{n}\right)
\end{aligned}
$$

which is also a normal-gamma distribution:

$$
P(\tilde{\mu}, \tilde{\tau} \mid D)=N G\left(\tilde{\mu}, \tilde{\tau} \mid \tilde{\mu}_{n}, \tilde{\kappa}_{n}, \tilde{\alpha}_{n}, \tilde{\beta}_{n}\right)
$$

\footnotetext{
${ }^{2}$ Further we use $\sigma$ and $\tau$ parameters interchangeably.
} 
with the parameters

$$
\begin{aligned}
\tilde{\mu}_{n} & =\frac{\tilde{\kappa}_{0}}{\tilde{\kappa}_{0}+n} \tilde{\mu}_{0}+\frac{n}{\tilde{\kappa}_{0}+n} \bar{x} \\
\tilde{\kappa}_{n} & =\tilde{\kappa}_{0}+n \\
\tilde{\alpha}_{n} & =\tilde{\alpha}_{0}+n / 2 \\
\tilde{\beta}_{n} & =\tilde{\beta}_{0}+\frac{1}{2} \sum_{i=1}^{n}\left(\tilde{x}_{i}-\bar{x}\right)^{2}+\frac{\tilde{\kappa}_{0} n\left(\bar{x}-\tilde{\mu}_{0}\right)^{2}}{2\left(\tilde{\kappa}_{0}+n\right)}
\end{aligned}
$$

where $\bar{x}=\frac{1}{n} \sum_{i=1}^{n} \tilde{x}_{i}$ is the mean of sampled data. The posterior distribution for $\tilde{\tau}$ is obtained by integrating Equation 24 over $\mu$ (See [19]) -

$$
\begin{aligned}
& p\left(\tilde{\tau} \mid D, \tilde{\mu}_{0}, \tilde{\kappa}_{0}, \tilde{\alpha}, \tilde{\beta}\right) \propto \\
&\left.\operatorname{Gamma}\left(\tilde{\alpha}+n / 2, \tilde{\beta}+\frac{1}{2} \sum_{i=1}^{n}\left(\tilde{x}_{i}-\bar{x}\right)^{2}+\frac{\tilde{\kappa} \tilde{\kappa}_{0}}{2\left(\tilde{\kappa}+\tilde{\kappa}_{0}\right)}\left(\bar{x}-\tilde{\mu}_{0}\right)^{2}\right)\right)
\end{aligned}
$$

Given the updated parameters $\theta=\left(\alpha_{0}, \beta_{0}, \mu_{0}, \kappa_{0}\right)$ using the rules 28, the predictive distribution for a new data $x_{\text {new }}$ is

$$
\begin{aligned}
& p\left(x_{\text {new }} \mid \boldsymbol{x}, \mu, \kappa, \alpha, \beta\right)= \\
& \quad \int p\left(x_{\text {new }} \mid \mu, \tau\right) p\left(\tau \mid \boldsymbol{x}, \mu_{0}, \kappa_{0}, \alpha, \beta\right) d \tau
\end{aligned}
$$

where

$$
p\left(x_{\text {new }} \mid \mu, \tau\right)=\left(\frac{\tau}{2 \pi}\right)^{1 / 2} e^{-\frac{\tau}{2}(x-\mu)^{2}} d \tau
$$

and $p\left(\tau \mid \boldsymbol{x}, \mu_{0}, \kappa_{0}, \alpha, \beta\right)$ is given by 29 Integral 30 is a Pearson type VII distribution (Equation 32 which is equivalent of the non-standardized Student's t-distribution.

$$
p\left(x_{\text {new }}\right)=\frac{1}{\alpha B(m-1 / 2,1 / 2)}\left(1+\left(\frac{x_{n+1}-\lambda}{\alpha}\right)^{2}\right)^{-m}
$$

where

$$
\begin{aligned}
m & =\alpha_{0}+(n+1) / 2 \\
\alpha & =A \sqrt{\sum_{i=1}^{n} x_{i}^{2}+\kappa_{0} \mu_{0}^{2}-\frac{\left(\sum_{i=1}^{n} x_{i}+\mu_{0} \kappa_{0}\right)^{2}}{n+\kappa_{0}}+2 \beta_{0}} \\
A & =\sqrt{\frac{n+1+\kappa_{0}}{n+\kappa_{0}}} \\
\lambda & =\frac{\sum_{i=1}^{n} x_{i}+\mu_{0} \kappa_{0}}{n+\kappa_{0}}
\end{aligned}
$$

Predictive distribution 4 in case of this data model is given by Equation 32 Please see detailed calculations in the Appendix.

\section{BLPA CHANGE DETECTOR}

The BLPA method is a combination of $\mathrm{BD}$ detector and PCCF predictive function. Particularly when we compute the joint probability $P\left(r_{t},\left\langle x_{j}\right\rangle_{j=1}^{t}\right)$ and after that when computing the run-length distribution $P\left(r_{t} \mid\left\langle x_{j}\right\rangle_{j=1}^{t}\right)$ we multiply these probabilities by the prior probability of the changes given by PCCF for the moment $t$. The BLPA method is depicted in Algorithm 1, in which:

- Lines 1-4: Set initial parameters values for the probability distribution of the data $D$.
- Line 5: Compute PCCF using initial values of the parameters (Equation 9).

- Line 7: Collect a new measurement.

- Line 8: Compute predictive distribution using Equation 32

- Line 9-10: Compute change probabilities and 'growth' probabilities of the run length.

- Line 11: Compute posterior probabilities of run lengths (changes).

- Line 12: Update parameters of the probability distributions for the data $D$ using Equations 28 .

- Lines 13-16: Find the most likely position of the last changepoint, update PCCF parameters and recalculate PCCF.

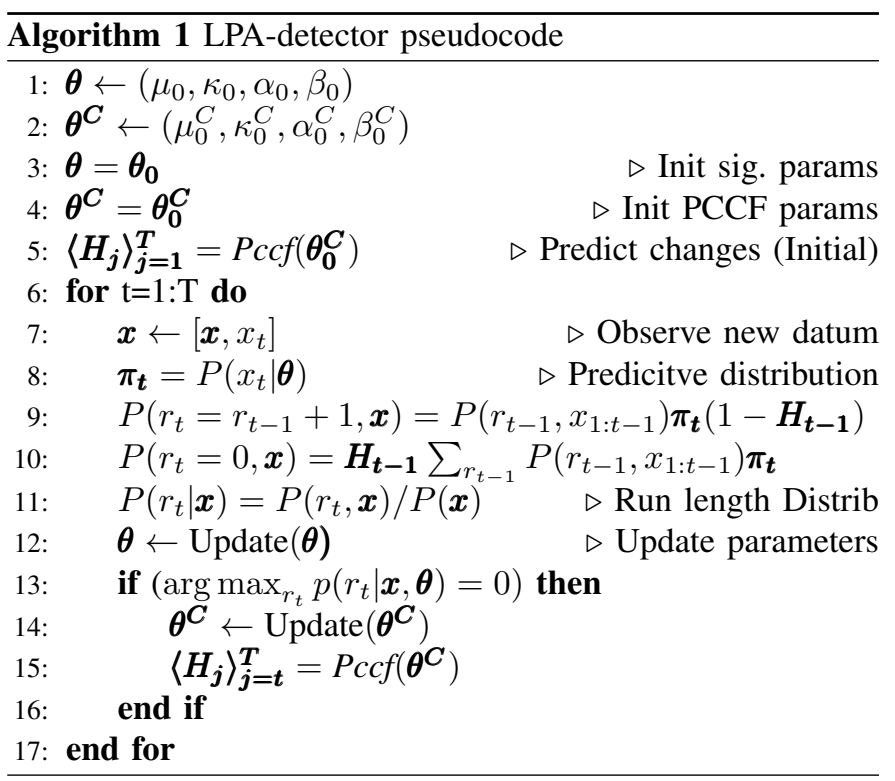

\section{EXPERIMENTS}

We performed experiments with artificially generated and real data sets. To measure the performance of the change detector we can consider it as as a binary classifier assigning labels 'change'/"not change' to the incoming observations $x_{t}$. If $\mathbf{e}_{t}^{+}$is the 'change' label assigned at the moment $t$ and $\mathbf{e}_{t}^{-}$is the label 'not change' assigned at $t$ then Then True Positive (TP), False Positives (FP), True Negatives (TN) and False Negatives (FN) events can be defined as follows:

- $\mathbf{e}_{t}^{+}$is TP if $\exists c_{i}: t-c_{i}<\delta$, and FP if $\nexists c_{i}: t-c_{i}<\delta$

- $\mathbf{e}_{t}^{-}$is FN if $\exists c_{i}: t-c_{i}<\delta$, and TN if $\nexists c_{i}: t-c_{i}<\delta$

The performance of the change detector is defined by TP/FP rates and by the average delay $\delta$ of the detection.

\section{A. Artificial data}

In the simulation we generated 200 signals with 10 recurrent changes in the mean value for each hazard-rate value $h$ varied in the interval from 50 to 300 by the step 15 . Average distance between changes was set to $\mu=100$ with the standard deviation $\sigma=10$. Results are depicted in Fig. 3. FP rate 


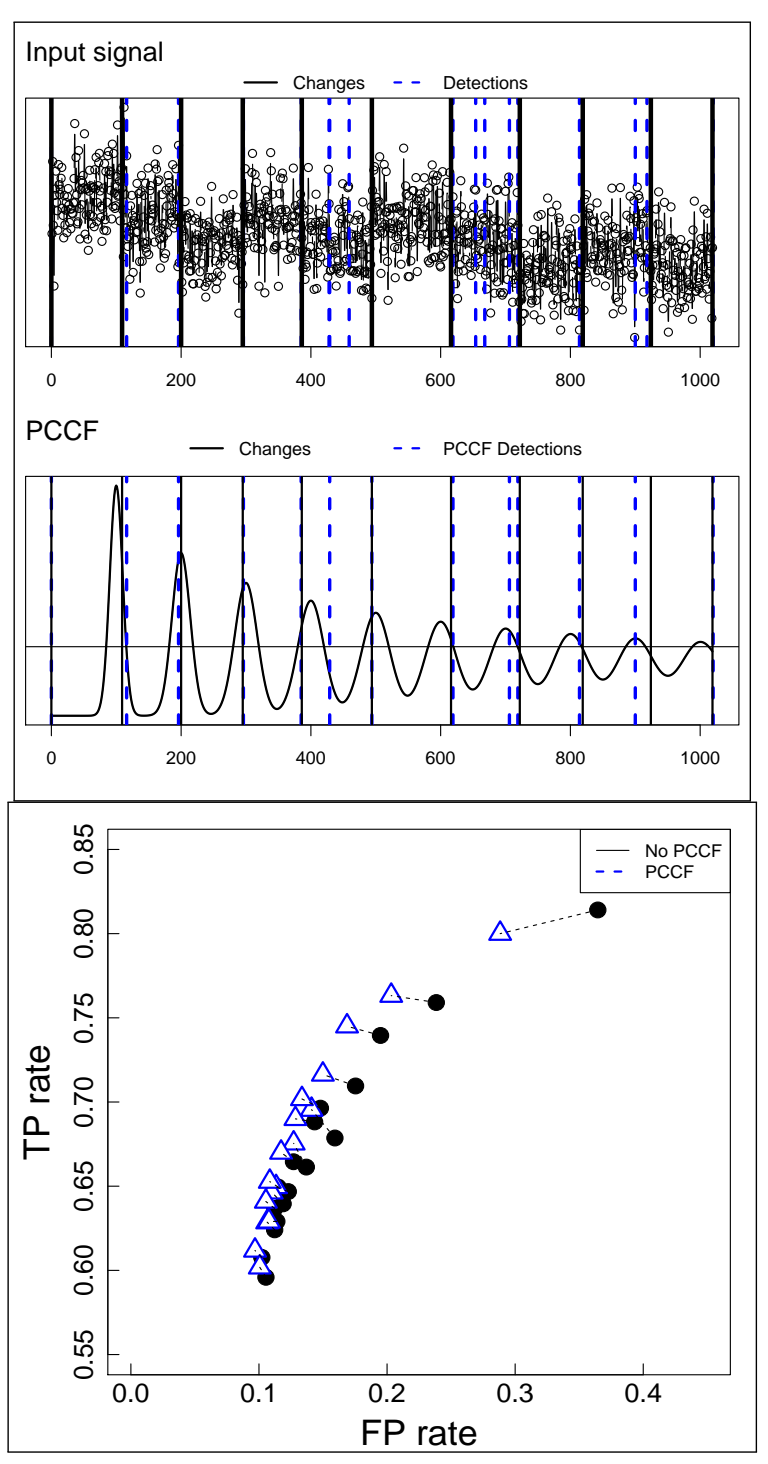

Fig. 3. Experimental results for simulated data streams with recurrent changes. Fig. 4. Experimental results for the 'Activity recognition' signal. On the On the top plot - an example of the generated input signal. Vertical solid top plot - illustrating example of the signal and corresponding PCCF function.

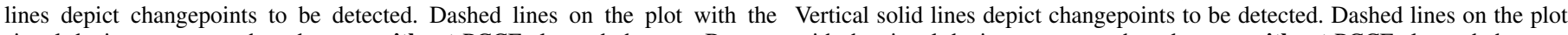
signal depict moments when detector without PCCF alarmed changes. Bottom with the signal depict moments when detector without PCCF alarmed changes.

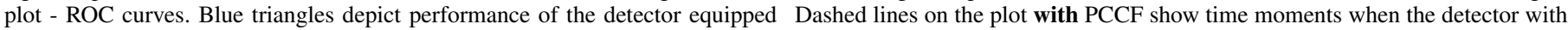

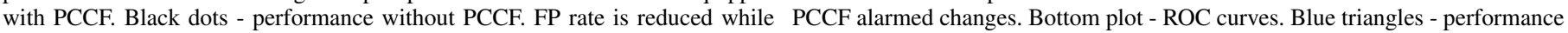
keeping the same TP rate.

is decreased while not reducing TP rate. In the worst cases the performance of both detectors is similar.

\section{B. Human Activity (HA) signal}

In the second experiment we used the Human activity data set [20] which contains sensor measurements from people performed 6 types of activities: three static postures (standing, sitting, lying) and three dynamic activities (walking, walking downstairs and walking upstairs). We detected changes in the signal caused by transitions from one set activities to another. Results are depicted in Fig. 4 FP rate is decreased when BD detector is used with the PCCF function.
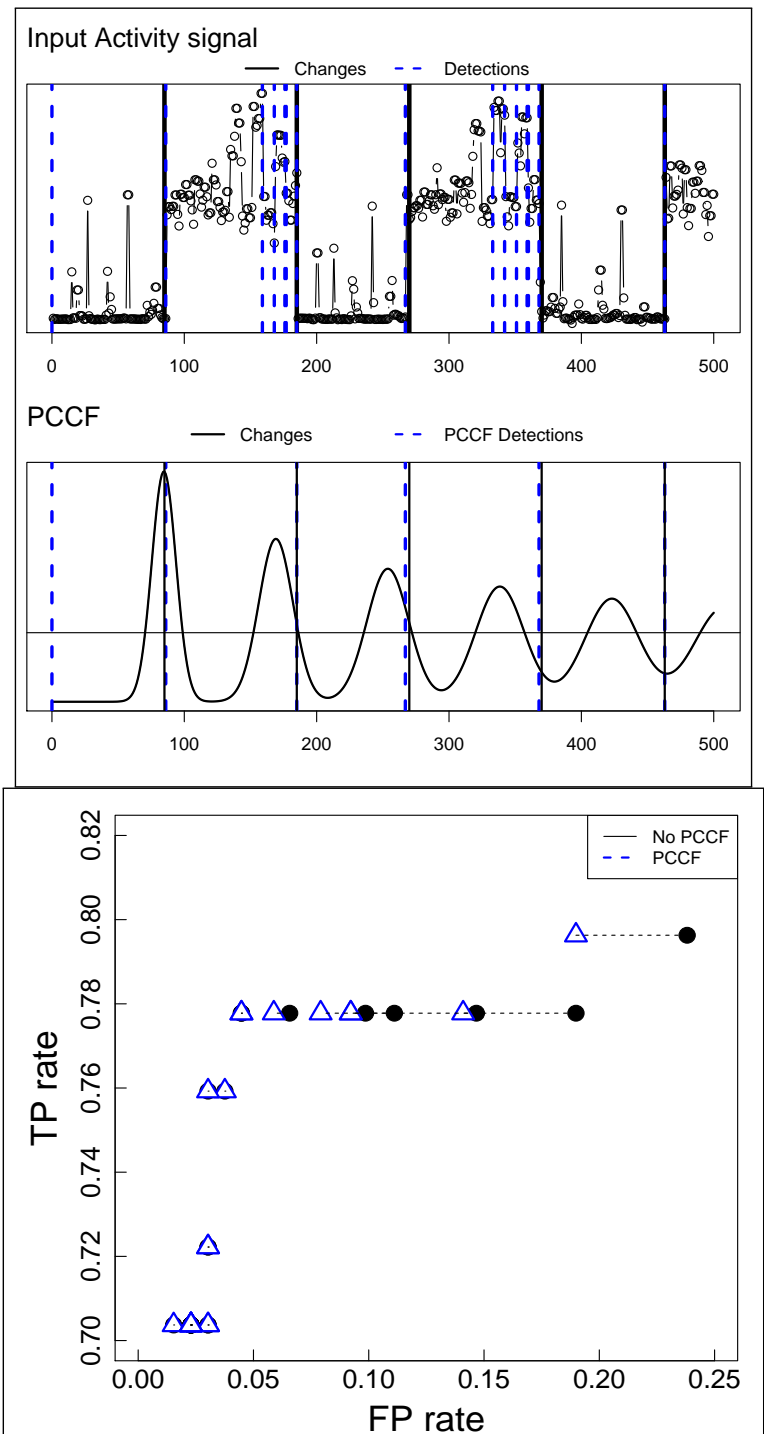

\section{CONCLUSION}

We proposed the method to improve performance of the Bayesian Online Changepoint detector (BD) for the data streams with recurrent changes by embedding into it the Predictive Confidence Change Function (PCCF). While observing a new data both BD detector's and PCCF's parameters are adjusted in a uniform way to the changing conditions using the same Bayesian update procedures constituting a two-layer adaptive change detection/prediction method BLPA. In the experiments with real and artificial data sets we demonstrated that Bayesian detector equipped with PCCF performs better in terms of TP/FP rates than the detector without PCCF. 


\section{REFERENCES}

[1] I. V. Nikiforov and M. Basseville, "Detection of Abrupt Changes," 1993.

[2] G. Widmer and M. Kubat, "Learning in the presence of concept drift and hidden contexts," Mach. Learn., vol. 23, no. 1, pp. 69-101, 1996.

[3] J. Gama, I. Zliobaite, A. Bifet, M. Pechenizkiy, and A. Bouchachia, "A survey on concept drift adaptation," ACM Computing Surveys, vol. 46, no. 4, pp. 44:1-44:37, 2014.

[4] D. J. M. Ryan Prescott Adams, "Bayesian online changepoint detection," 2007.

[5] A. Maslov, M. Pechenizkiy, I. Žliobaitė, and T. Kärkkäinen, "Modelling recurrent events for improving online change detection," in SIAM International Conference on Data Mining (SDM16), 2016.

[6] A. S. Polunchenko and A. G. Tartakovsky, "State-of-the-Art in Sequential Change-Point Detection," Methodology and Computing in Applied Probability, vol. 14, no. 3, pp. 649-684, Oct. 2011.

[7] R. C. Wilson, M. R. Nassar, and J. I. Gold, "Bayesian online learning of the hazard rate in change-point problems," Neural computation, vol. 22, no. 9, pp. 2452-2476, 2010.

[8] D. Huang, Y. S. Koh, G. Dobbie, and R. Pears, "Detecting volatility shift in data streams," in ICDM'2014, pp. 863-868.

[9] R. C. Wilson, M. R. Nassar, and J. I. Gold, "Bayesian online learning of the hazard rate in change-point problems," Neural Comput., vol. 22, no. 9, pp. 2452-2476, Sep. 2010. [Online]. Available: http://dx.doi.org/10.1162/NECO_a_00007

[10] A. B. Downey, "A novel changepoint detection algorithm," 2008. [Online]. Available: http://arxiv.org/abs/0812.1237v1

[11] Y. Saatçi, R. D. Turner, and C. E. Rasmussen, "Gaussian process change point models," in Proceedings of the 27th International Conference on Machine Learning (ICML-10), 2010, pp. 927-934.

[12] J. Gama and P. Kosina, "Learning about the learning process," in Proc. of IDA'11, pp. 162-172.

[13] J. B. Gomes, M. M. Gaber, P. A. C. Sousa, and E. M. Ruiz, "Mining recurring concepts in a dynamic feature space," IEEE Trans. Neural Netw. Learning Syst., vol. 25, no. 1, pp. 95-110, 2014.

[14] J. B. Gomes, P. A. C. Sousa, and E. M. Ruiz, "Tracking recurrent concepts using context," Intell. Data Anal., vol. 16, no. 5, pp. 803-825.

[15] H. Ang, V. Gopalkrishnan, I. Zliobaite, M. Pechenizkiy, and S. Hoi, "Predictive handling of asynchronous concept drifts in distributed environments," IEEE Trans. on Knowl. and Data Eng., vol. 25, pp. 23432355, 2013.

[16] I. Zliobaite, "Change with delayed labeling: When is it detectable?" in ICDM 2010 Workshops, pp. 843-850.

[17] D. R. Cox, Renewal theory. Methuen, 1962, vol. 58.

[18] W. Feller, An introduction to probability theory and its applications: volume I. John Wiley \& Sons London-New York-Sydney-Toronto, 1968, vol. 3 .

[19] M. I. Jordan, "Chapter 9. the exponential family: Conjugate priors." [Online]. Available: https://people.eecs.berkeley.edu/ jordan/courses/ 260-spring10/other-readings/

[20] J.-L. Reyes-Ortiz, L. Oneto, A. Samà, X. Parra, and D. Anguita, "Transition-aware human activity recognition using smartphones," Neurocomputing, vol. 171, pp. 754-767, 2016.

\section{APPENDIX}

Assuming Gaussian distribution

$$
p(x \mid \mu, \tau)=\frac{\sqrt{\tau}}{\sqrt{2 \pi}} e^{-\frac{\tau}{2}(x-\mu)^{2}}
$$

Probability $p\left(x_{n+1} \mid x_{1}, \ldots, x_{n}\right)$ can be found as

$$
\begin{aligned}
& p\left(x_{n+1} \mid x_{1}, \ldots, x_{n}\right) \\
& =\frac{\int_{\tau \in \mathbb{R}^{+}} \int_{\mu \in \mathbb{R}} p\left(x_{1}, \ldots, x_{n+1} \mid \mu, \tau\right) p(\mu, \tau) d \mu d \tau}{\int_{\tau \in \mathbb{R}^{+}} \int_{\mu \in \mathbb{R}} p\left(x_{1}, \ldots, x_{n} \mid \mu, \tau\right) p(\mu, \tau) d \mu d \tau} .
\end{aligned}
$$

The value $p\left(x_{1}, \ldots, x_{n} \mid \mu, \tau\right) p(\mu, \tau)$ is

$$
\begin{aligned}
\left(\frac{\sqrt{\tau}}{\sqrt{2 \pi}}\right)^{n} \times & \exp \left(-\frac{\tau}{2} \sum_{i=1}^{n}\left(x_{i}-\mu\right)^{2}\right) \tau^{\alpha_{0}-1 / 2} \\
& \times \exp \left(-\frac{\tau}{2}\left(\kappa_{0}\left(\mu-\mu_{0}\right)^{2}+2 \beta_{0}\right)\right) .
\end{aligned}
$$

An expression in the exponent is equivalent to

$$
\begin{aligned}
& -\frac{\tau\left(n+\kappa_{0}\right)}{2} \sum_{i=1}^{n}\left(\mu-\frac{\sum_{i=1}^{n} x_{i}+\mu_{0} \kappa_{0}}{n+\kappa_{0}}\right)^{2} \\
& -\frac{\tau}{2}\left(\sum_{i=1}^{n} x_{i}^{2}+\kappa_{0} \mu_{0}^{2}-\frac{\left(\sum_{i=1}^{n} x_{i}+\mu_{0} \kappa_{0}\right)^{2}}{n+\kappa_{0}}+2 \beta_{0}\right),
\end{aligned}
$$

from where

$$
\begin{aligned}
& p\left(x_{1}, \ldots, x_{n} \mid \mu, \tau\right) p(\mu, \tau) \\
&=\left(\frac{1}{\sqrt{2 \pi}}\right)^{n-1} \frac{\Gamma\left(\alpha_{0}+n / 2\right)}{\sqrt{n+\kappa_{0}}}\left(\frac{\hat{b}_{n}}{2}\right)^{-\alpha_{0}+n / 2} \\
& \times p_{G a m m a}\left(\alpha_{0}+n / 2, \hat{a}_{n} / 2\right) \\
&
\end{aligned}
$$

where

$$
\begin{aligned}
& \hat{a}_{n}=\left(\sum_{i=1}^{n} x_{i}^{2}+\kappa_{0} \mu_{0}^{2}-\frac{\left(\sum_{i=1}^{n} x_{i}+\mu_{0} \kappa_{0}\right)^{2}}{n+\kappa_{0}}+2 \beta_{0}\right), \\
& \hat{\mu}_{n}=\frac{\sum_{i=1}^{n} x_{i}+\mu_{0} \kappa_{0}}{n+\kappa_{0}}, \hat{\sigma}_{n}^{2}=\frac{1}{\tau\left(n+\kappa_{0}\right)} .
\end{aligned}
$$

Therefore

$$
\begin{aligned}
& \int_{\tau \in \mathbb{R}^{+}} \int_{\mu \in \mathbb{R}} p\left(x_{1}, \ldots, x_{n+1} \mid \mu, \tau\right) p(\mu, \tau) d \mu d \tau \\
= & \left(\frac{1}{\sqrt{2 \pi}}\right)^{n-1} \frac{\Gamma\left(\alpha_{0}\right)}{\sqrt{n+\kappa_{0}}}\left(\frac{\hat{a}_{n}}{2}\right)^{-\left(\alpha_{0}+n / 2\right)}
\end{aligned}
$$

and integral 37 can be expressed as

$$
\begin{aligned}
& \frac{\sqrt{n+\kappa_{0}}}{\sqrt{n+1+\kappa_{0}} B\left(\alpha_{0}+n / 2,1 / 2\right)} \frac{\hat{a}_{n}^{\alpha_{0}+n / 2}}{\hat{a}_{n+1}^{\alpha_{0}+(n+1) / 2}} \\
= & \frac{\sqrt{n+\kappa_{0}}}{\sqrt{n+1+\kappa_{0}} B\left(\alpha_{0}+n / 2,1 / 2\right)}\left(\frac{\hat{a}_{n+1}}{\hat{a}_{n}}\right)^{-\left(\alpha_{0}+(n+1) / 2\right)} \hat{a}_{n}^{-1 / 2} .
\end{aligned}
$$

Noting that

$$
\begin{aligned}
& \frac{\hat{a}_{n+1}}{\hat{a}_{n}}=1+\frac{n+\kappa_{0}}{\hat{a}_{n}\left(n+1+\kappa_{0}\right)} \\
\times & \left(x_{n+1}^{2}-\frac{2 x_{n+1}\left(\sum_{i=1}^{n} x_{i}+\mu_{0} \kappa_{0}\right)}{n+\kappa_{0}}+\frac{\left(\sum_{i=1}^{n} x_{i}+\mu_{0} \kappa_{0}\right)^{2}}{\left(n+\kappa_{0}\right)^{2}}\right)
\end{aligned}
$$

from where

$$
\begin{aligned}
& p\left(x_{n+1} \mid x_{1}, \ldots, x_{n}\right) \\
= & \frac{1}{\hat{b}_{n} B\left(\alpha_{0}+n / 2,1 / 2\right)}\left(1+\frac{\left(x_{n+1}-\lambda\right)^{2}}{\hat{b}_{n}^{2}}\right)^{-\left(\alpha_{0}+(n+1) / 2\right)},
\end{aligned}
$$

where coefficients are

$$
\hat{b}_{n}=\frac{\sqrt{\left(n+1+\kappa_{0}\right) \hat{a}_{n}}}{\sqrt{\left(n+\kappa_{0}\right)}}, \lambda=\frac{\sum_{i=1}^{n} x_{i}+\mu_{0} \kappa_{0}}{n+\kappa_{0}}
$$

